Research Article

\title{
New Iterative Scheme for Finite Families of Equilibrium, Variational Inequality, and Fixed Point Problems in Banach Spaces
}

\author{
Shenghua Wang ${ }^{1,2}$ and Caili Zhou ${ }^{3}$ \\ ${ }^{1}$ School of Applied Mathematics and Physics, North China Electric Power University, \\ Baoding 071003, China \\ ${ }^{2}$ Department of Mathematics, Gyeongsang National University, Jinju 660-714, Republic of Korea \\ ${ }^{3}$ College of Mathematics and Computer, Hebei University, Baoding 071002, China
}

Correspondence should be addressed to Shenghua Wang, sheng-huawang@hotmail.com

Received 6 December 2010; Accepted 30 January 2011

Academic Editor: S. Al-Homidan

Copyright $\odot 2011 \mathrm{~S}$. Wang and C. Zhou. This is an open access article distributed under the Creative Commons Attribution License, which permits unrestricted use, distribution, and reproduction in any medium, provided the original work is properly cited.

We introduced a new iterative scheme for finding a common element in the set of common fixed points of a finite family of quasi- $\phi$-nonexpansive mappings, the set of common solutions of a finite family of equilibrium problems, and the set of common solutions of a finite family of variational inequality problems in Banach spaces. The proof method for the main result is simplified under some new assumptions on the bifunctions.

\section{Introduction}

Throughout this paper, let $\mathbb{R}$ denote the set of all real numbers. Let $E$ be a smooth Banach space and $E^{*}$ the dual space of $E$. The function $\phi: E \times E \rightarrow \mathbb{R}$ is defined by

$$
\phi(x, y)=\|x\|^{2}-\langle y, J x\rangle+\|y\|^{2}, \quad \forall x, y \in E,
$$

where $J$ is the normalized dual mapping from $E$ to $E^{*}$ defined by

$$
J(x)=\left\{x^{*} \in E^{*}:\left\langle x, x^{*}\right\rangle=\|x\|^{2}=\left\|x^{*}\right\|^{2}\right\}, \quad \forall x \in E .
$$


Let $C$ be a nonempty closed and convex subset of $E$. The generalized projection $\Pi: E \rightarrow C$ is a mapping that assigns to an arbitrary point $x \in E$ the minimum point of the function $\phi(x, y)$, that is, $\Pi_{C} x=\widehat{x}$, where $\widehat{x}$ is the solution to the minimization problem

$$
\phi(\widehat{x}, x)=\inf _{z \in C} \phi(z, x)
$$

In Hilbert spaces, $\phi(x, y)=\|x-y\|^{2}$ and $\Pi_{C}=P_{C}$, where $P_{C}$ is the metric projection. It is obvious from the definition of function $\phi$ that

$$
(\|y\|-\|x\|)^{2} \leq \phi(y, x) \leq(\|y\|+\|x\|)^{2}, \quad \forall x, y \in E .
$$

We remark that if $E$ is a reflexive, strictly convex and smooth Banach space, then for $x, y \in E$, $\phi(x, y)=0$ if and only if $x=y$. For more details on $\phi$ and $\Pi$, the readers are referred to [1-4].

Let $T$ be a mapping from $C$ into itself. We denote the set of fixed points of $T$ by $F(T)$. $T$ is called to be nonexpansive if $\|T x-T y\| \leq\|x-y\|$ for all $x, y \in C$ and quasi-nonexpansive if $F(T) \neq \emptyset$ and $\|x-T y\| \leq\|x-y\|$ for all $x \in F(T)$ and $y \in C$. A point $p \in C$ is called to be an asymptotic fixed point of $T$ [5] if $C$ contains a sequence $\left\{x_{n}\right\}$ which converges weakly to $p$ such that $\lim _{n \rightarrow \infty}\left\|x_{n}-T x_{n}\right\|=0$. The set of asymptotic fixed points of $T$ is denoted by $\widehat{F}(T)$. The mapping $T$ is said to be relatively nonexpansive [6-8] if $\widehat{F}(T)=F(T)$ and $\phi(p, T x) \leq$ $\phi(p, x)$ for all $x \in C$ and $p \in F(T)$. The mapping $T$ is said to be $\phi$-nonexpansive if $\phi(T x, T y) \leq$ $\phi(x, y)$ for all $x, y \in C$. T is called to be quasi- $\phi$-nonexpansive [9] if $F(T) \neq \emptyset$ and $\phi(p, T x) \leq$ $\phi(p, x)$ for all $x \in C$ and $p \in F(T)$.

In 2005, Matsushita and Takahashi [10] introduced the following algorithm:

$$
\begin{gathered}
x_{0}=x \in C, \\
y_{n}=J^{-1}\left(\alpha_{n} J x_{n}+\left(1-\alpha_{n}\right) J T x_{n}\right), \\
C_{n}=\left\{z \in C: \phi\left(z, y_{n}\right) \leq \phi\left(z, x_{n}\right)\right\}, \\
Q_{n}=\left\{z \in C:\left\langle x_{n}-z, J x-J x_{n}\right\rangle \geq 0\right\}, \\
x_{n+1}=P_{C_{n} \cap Q_{n}} x, \quad \forall n \geq 0,
\end{gathered}
$$

where $J$ is the duality mapping on $E, T$ is a relatively nonexpansive mapping from $C$ into itself, and $\left\{\alpha_{n}\right\}$ is a sequence of real numbers such that $0 \leq \alpha_{n}<1$ and $\lim _{\sup _{n \rightarrow \infty}} \alpha_{n}<1$ and proved that the sequence $\left\{x_{n}\right\}$ generated by (1.5) converges strongly to $P_{F(T)} x$, where $P_{F(T)}$ is the generalized projection from $C$ onto $F(T)$.

Let $f$ be a bifunction from $C \times C$ to $\mathbb{R}$. The equilibrium problem for $f$ is to find $p \in C$ such that

$$
f(p, y) \geq 0, \quad \forall y \in C
$$

We use $\operatorname{EP}(f)$ to denote the solution set of the equilibrium problem (1.6). That is,

$$
\operatorname{EP}(f)=\{p \in C: f(p, y) \geq 0, \forall y \in C\}
$$


For studying the equilibrium problem, $f$ is usually assumed to satisfy the following conditions:

(A1) $f(x, x)=0$ for all $x \in C$;

(A2) $f$ is monotone, that is, $f(x, y)+f(y, x) \leq 0$ for all $x, y \in C$;

(A3) for each $x, y, z \in C, \lim \sup _{t \rightarrow 0} f(t z+(1-t) x, y) \leq f(x, y)$;

(A4) for each $x \in C, y \rightarrow f(x, y)$ is convex and lower semicontinuous.

Recently, many authors investigated the equilibrium problems in Hilbert spaces or Banach spaces; see, for example, [11-25]. In [20], Qin et al. considered the following iterative scheme by a hybrid method in a Banach space:

$$
\begin{gathered}
x_{0} \in E \quad \text { chosen arbitrarily, } \\
C_{1}=C, \\
x_{1}=\Pi_{C_{1}} x_{0}, \\
y_{n}=J^{-1}\left(\alpha_{n, 0} J x_{n}+\sum_{i=1}^{N} \alpha_{n, i} J T_{i} x_{n}\right), \\
u_{n} \in C \quad \text { such that } f\left(u_{n}, y\right)+\frac{1}{r_{n}}\left\langle y-u_{n} J u_{n}-J y_{n}\right\rangle \geq 0, \quad \forall y \in C, \\
C_{n+1}=\left\{z \in C_{n}: \phi\left(z, u_{n}\right) \leq \phi\left(z, x_{n}\right)\right\}, \\
x_{n+1}=\prod_{C_{n+1}} x_{0},
\end{gathered}
$$

where $T_{i}: C \rightarrow C$ is a closed quasi- $\phi$-nonexpansive mapping for each $i \in\{1,2, \ldots, N\}$, $\alpha_{n, 0},\left\{\alpha_{n, 1}\right\}, \ldots,\left\{\alpha_{n, N}\right\}$ are real sequences in $(0,1)$ satisfying $\sum_{j=0}^{N} \alpha_{n, j}=1$ for each $n \geq 1$ and $\liminf _{n \rightarrow \infty} \alpha_{n, 0} \alpha_{n, i}>0$ for each $i \in\{1,2, \ldots, N\}$ and $\left\{r_{n}\right\}$ is a real sequence in $[a, \infty)$ with $a>0$. Then the authors proved that $\left\{x_{n}\right\}$ converges strongly to $\prod_{\mathcal{F}} x_{0}$, where $\mathcal{F}=\bigcap_{i=1}^{N} F\left(T_{i}\right) \cap \mathrm{EP}(f)$.

Very recently, Zegeye and Shahzad [25] introduced a new scheme for finding an element in the common fixed point set of finite family of closed relatively quasi-nonexpansive mappings, common solutions set of finite family of equilibrium problems, and common solutions set of finite family of variational inequality problems for monotone mappings in a Banach space. More precisely, let $f_{i}: C \times C \rightarrow \mathbb{R}, i=1,2, \ldots, L$, be a finite family of bifunctions, $S_{j}: C \rightarrow C, j=1, \ldots, D$, a finite family of relatively quasi-nonexpansive mappings, and $A_{i}: \mathrm{C} \rightarrow E^{*}, i=1,2, \ldots, N$, a finite family of continuous monotone mappings. For $x \in E$, define the mappings $F_{r_{n}}, T_{r_{n}}: E \rightarrow C$ by

$$
\begin{gathered}
F_{r_{n}} x=\left\{z \in C:\left\langle y-z, A_{n} z\right\rangle+\frac{1}{r_{n}}\langle y-z, J z-J x\rangle \geq 0, \forall y \in C\right\}, \\
T_{r_{n}} x=\left\{z \in C: f_{n}(z, y)+\frac{1}{r_{n}}\langle y-z, J z-J x\rangle \geq 0, \forall y \in C\right\},
\end{gathered}
$$


where $A_{n}=A_{n(\bmod N)}, f_{n}=f_{n(\bmod L)}$ and $r_{n} \subset\left[c_{1}, \infty\right)$ for some $c_{1}>0$. Zegeye and Shahzad [25] introduced the following scheme:

$$
\begin{gathered}
x_{0} \in C_{0}=C \text { chosen arbitrarily, } \\
z_{n}=F_{r_{n}} x_{n}, \\
u_{n}=T_{r_{n}} x_{n}, \\
y_{n}=J^{-1}\left(\alpha_{0} J x_{n}+\alpha_{1} J z_{n}+\alpha_{2} J S_{n} u_{n}\right), \\
C_{n+1}=\left\{z \in C_{n}: \phi\left(z, y_{n}\right) \leq \phi\left(z, x_{n}\right)\right\}, \\
x_{n+1}=\prod_{C_{n+1}} x_{0},
\end{gathered}
$$

where $S_{n}=S_{n(\bmod D)}, \alpha_{0}, \alpha_{1}, \alpha_{2} \in(0,1)$ such that $\alpha_{0}+\alpha_{1}+\alpha_{2}=1$. Further, they proved that $\left\{x_{n}\right\}$ converges strongly to an element of $\mathcal{F}$, where $\mathcal{F}=\left[\bigcap_{j=1}^{D} F\left(S_{j}\right)\right] \cap\left[\bigcap_{i=1}^{N} \operatorname{VI}\left(C, A_{i}\right)\right] \cap$ $\left[\bigcap_{l=1}^{L} \operatorname{EP}\left(f_{l}\right)\right]$.

In this paper, motivated and inspired by the iterations (1.8) and (1.10), we consider a new iterative process with a finite family of quasi- $\phi$-nonexpansive mappings for a finite family of equilibrium problems and a finite family of variational inequality problems in a Banach space. More precisely, let $\left\{S_{i}\right\}_{i=1}^{N_{1}}: C \rightarrow C$ be a family of quasi- $\phi$-nonexpansive mappings, $\left\{f_{i}\right\}_{i=1}^{N_{2}}: C \times C \rightarrow \mathbb{R}$ a finite family of bifunctions, and $\left\{A_{i}\right\}_{i=1}^{N_{3}}: C \rightarrow E^{*}$ a finite family of continuous monotone mappings such that $\mathcal{F}=\left[\bigcap_{i=1}^{N_{1}} F\left(S_{i}\right)\right] \cap\left[\bigcap_{i=1}^{N_{2}} \operatorname{EP}\left(f_{i}\right)\right] \cap$ $\left[\bigcap_{i=1}^{N_{3}} \operatorname{VI}\left(C, A_{i}\right)\right] \neq \emptyset$. Let $\left\{r_{1, i}\right\}_{i=1}^{N_{2}} \subset(0, \infty)$ and $\left\{r_{2, i}\right\}_{i=1}^{N_{3}} \subset(0, \infty)$. Define the mappings $T_{r_{1, i}}$, $F_{r_{2, i}}: E \rightarrow C$ by

$$
\begin{gathered}
T_{r_{1, i}} x=\left\{z \in C: f_{i}(z, y)+\frac{1}{r_{1, i}}\langle y-z, J z-J x\rangle \geq 0, \forall y \in C\right\}, \quad i=1, \ldots, N_{2}, \\
F_{r_{2, i}} x=\left\{z \in C:\left\langle y-z, A_{i} z\right\rangle+\frac{1}{r_{2, i}}\langle y-z, J z-J x\rangle \geq 0, \forall y \in C\right\}, \quad i=1, \ldots, N_{3} .
\end{gathered}
$$

Consider the iteration

$$
\begin{gathered}
x_{1} \in C \text { chosen arbitrarily, } \\
y_{n}=J^{-1}\left(\alpha_{0} J x_{n}+\alpha_{1} \sum_{i=1}^{N_{1}} \lambda_{1, i} J S_{i} x_{n}+\alpha_{2} \sum_{i=1}^{N_{2}} \lambda_{2, i} J T_{r_{1, i}} x_{n}+\alpha_{3} \sum_{i=1}^{N_{3}} \lambda_{3, i} J F_{r_{2, i}} x_{n}\right) \\
C_{n}=\left\{v \in C: \phi\left(v, y_{n}\right) \leq \phi\left(v, x_{n}\right)\right\} \\
D_{n}=\bigcap_{i=1}^{n} C_{i} \\
x_{n+1}=\prod_{D_{n}} x_{1}, \quad n \geq 1
\end{gathered}
$$

where $\alpha_{0}, \alpha_{1}, \alpha_{2}, \alpha_{3}$ are the real numbers in $(0,1)$ satisfying $\alpha_{0}+\alpha_{1}+\alpha_{2}+\alpha_{3}=1$ and for each $j=1,2,3, \lambda_{j, 1}, \ldots, \lambda_{j, N_{j}}$ are the real numbers in $(0,1)$ satisfying $\sum_{i=1}^{N_{j}} \lambda_{j, i}=1$. We will prove that 
the sequence $\left\{x_{n}\right\}$ generated by (1.13) converges strongly to an element in $\mathcal{F}$. In this paper, in order to simplify the proof, we will replace the condition (A3) with (A3'): for each fixed $y \in C, f(\cdot, y)$ is continuous.

Obviously, the condition (A3') implies (A3). Under the condition (A3'), we will show that each $T_{r_{1, i}}$ (as well as $F_{r_{2, j}}, i=1, \ldots, N_{2}, j=1, \ldots, N_{3}$ ) is closed which is such that the proof for the main result of this paper is simplified.

\section{Preliminaries}

The modulus of smoothness of a Banach space $E$ is the function $\rho_{E}:[0, \infty) \rightarrow[0, \infty)$ defined by

$$
\rho_{E}(\tau)=\sup \left\{\frac{\|x+y\|+\|x-y\|}{2}-1:\|x\|=1 ;\|y\|=\tau\right\}
$$

The space $E$ is said to be smooth if $\rho_{E}(\tau)>0$, for all $\tau>0$, and $E$ is called uniformly smooth if and only if $\lim _{\tau \rightarrow 0^{+}} \rho_{E}(\tau) / \tau=0$.

A Banach space $E$ is said to be strictly convex if $\|(x+y) / 2\|<1$ for all $x, y \in E$ with $\|x\|=\|y\|=1$ and $x \neq y$. It is said to be uniformly convex if $\lim _{n \rightarrow \infty}\left\|x_{n}-y_{n}\right\|=0$ for any two sequences $\left\{x_{n}\right\}$ and $\left\{y_{n}\right\}$ in $E$ such that $\left\|x_{n}\right\|=\left\|y_{n}\right\|=1$ and $\lim _{n \rightarrow \infty}\left\|\left(x_{n}+y_{n}\right) / 2\right\|=1$. It is known that if a Banach space $E$ is uniformly smooth, then its dual space $E^{*}$ is uniformly convex.

A Banach space $E$ is called to have the Kadec-Klee property if for any sequence $\left\{x_{n}\right\} \subset$ $E$ and $x \in E$ with $x_{n} \rightarrow x$, where $\rightarrow$ denotes the weak convergence, and $\left\|x_{n}\right\| \rightarrow\|x\|$, then $x_{n}-x \rightarrow 0$ as $n \rightarrow \infty$, where $\rightarrow$ denotes the strong convergence. It is well known that every uniformly convex Banach space has the Kadec-Klee property. For more details on the Kadec-Klee property, the reader is referred to $[3,4]$.

Let $C$ be a nonempty closed and convex subset of a Banach space $E$. A mapping $S$ : $C \rightarrow C$ is said to be closed if for any sequence $\left\{x_{n}\right\} \subset C$ such that $\lim _{n \rightarrow \infty} x_{n}=x_{0}$ and $\lim _{n \rightarrow \infty} S x_{n}=y_{0}, S x_{0}=y_{0}$.

Let $A: D(A) \subset E \rightarrow E^{*}$ be a mapping. $A$ is said to be monotone if for each $x, y \in D(A)$, the following inequality holds:

$$
\langle x-y, A x-A y\rangle \geq 0 \text {. }
$$

Let $A$ be a monotone mapping from $C$ into $E^{*}$. The variational inequality problem on $A$ is formulated as follows:

$$
\text { find a point } u \in C \text { such that }\langle v-u, A u\rangle \geq 0, \quad \forall v \in C \text {. }
$$

The solution set of the above variational inequality problem is denoted by $\operatorname{VI}(C, A)$. 
Next we state some lemmas which will be used later.

Lemma 2.1 (see [1]). Let $C$ be a nonempty closed and convex subset of a smooth Banach space E and $x \in E$. Then, $x_{0}=\Pi_{C} x$ if and only if

$$
\left\langle x_{0}-y, J x-J x_{0}\right\rangle \geq 0 \quad \forall y \in C
$$

Lemma 2.2 (see [1]). Let E be a reflexive, strictly convex and smooth Banach space, $C$ a nonempty closed and convex subset of $E$, and $x \in E$. Then

$$
\phi\left(y, \Pi_{C} x\right)+\phi\left(\Pi_{C} x, x\right) \leq \phi(y, x), \quad \forall y \in C .
$$

Lemma 2.3 (see [20]). Let $E$ be a strictly convex and smooth Banach space, $C$ a nonempty closed and convex subset of $E$, and $T: C \rightarrow C$ a quasi- $\phi$-nonexpansive mapping. Then $F(T)$ is a closed and convex subset of $C$.

Since the condition (A3') implies (A3), the following lemma is a natural result of [22, Lemmas 2.8 and 2.9].

Lemma 2.4. Let $C$ be a closed and convex subset of a smooth, strictly convex and reflexive Banach space $E$. Let $f$ be a bifunction from $C \times C \rightarrow \mathbb{R}$ satisfying (A1), (A2), (A3'), and (A4). Let $r>0$ and $x \in E$. Then

(a) there exists $z \in C$ such that

$$
f(z, y)+\frac{1}{r}\langle y-z, J z-J x\rangle \geq 0, \quad \forall y \in C
$$

(b) define a mapping $T_{r}: E \rightarrow C$ by

$$
T_{r} x=\left\{z \in C: f(z, y)+\frac{1}{r}\langle y-z, J z-J x\rangle \geq 0, \forall y \in C\right\} .
$$

Then the following conclusions hold:

(1) $T_{r}$ is single-valued;

(2) $T_{r}$ is firmly nonexpansive, that is, for all $x, y \in E$,

$$
\left\langle T_{r} x-T_{r} y, J T_{r} x-J T_{r} y\right\rangle \leq\left\langle T_{r} x-T_{r} y, J x-J y\right\rangle
$$

(3) $F\left(T_{r}\right)=\mathrm{EP}(f)$;

(4) $T_{r}$ is quasi- $\phi$-nonexpansive;

(5) $\mathrm{EP}(f)$ is closed and convex;

(6) $\phi\left(p, T_{r} x\right)+\phi\left(T_{r} x, x\right) \leq \phi(p, x)$, for all $p \in F\left(T_{r}\right)$. 
Remark 2.5. Let $A: C \rightarrow E^{*}$ be a continuous monotone mapping and define $f(x, y)=\langle y-$ $x, A x\rangle$ for all $x, y \in C$. It is easy to see that $f$ satisfies the conditions (A1), (A2), (A3'), and $(\mathrm{A} 4)$ and $\mathrm{EP}(f)=\operatorname{VI}(C, A)$. Hence, for every real number $r>0$, if defining a mapping $F_{r}$ : $E \rightarrow C$ by

$$
F_{r} x=\left\{z \in C:\langle y-z, A z\rangle+\frac{1}{r}\langle y-z, J z-J x\rangle \geq 0, \forall y \in C\right\}
$$

then $F_{r}$ satisfies all the conclusions in Lemma 2.4. See [25, Lemma 2.4].

Lemma 2.6 (see [26]). Let $p>1$ and $s>0$ be two fixed real numbers. Then a Banach space $E$ is uniformly convex if and only if there exists a continuous strictly increasing convex function $g:[0, \infty)$ with $g(0)=0$ such that

$$
\|\lambda x+(1-\lambda) y\|^{p} \leq \lambda\|x\|^{p}+(1-\lambda)\|y\|^{2}-w_{p}(\lambda) g(\|x-y\|)
$$

for all $x, y \in B_{s}(0)=\{x \in E:\|x\| \leq s\}$ and $\lambda \in[0,1]$, where $w_{p}(\lambda)=\lambda^{p}(1-\lambda)+\lambda(1-\lambda)^{p}$.

The following lemma can be obtained from Lemma 2.6 immediately; also see [20, Lemma 1.9].

Lemma 2.7 (see [20]). Let E be a uniformly convex Banach space, $s>0$ a positive number, and $B_{s}(0)$ a closed ball of $E$. There exists a continuous, strictly increasing and convex function $g:[0, \infty)$ with $g(0)=0$ such that

$$
\left\|\sum_{i=1}^{N} \alpha_{i} x_{i}\right\|^{2} \leq \sum_{i=1}^{N} \alpha_{i}\left\|x_{i}\right\|^{2}-\alpha_{j} \alpha_{k} g\left(\left\|x_{j}-x_{k}\right\|\right), \quad j, k \in\{1,2, \ldots, N\} \text { with } j \neq k
$$

for all $x_{1}, x_{2}, \ldots, x_{N} \in B_{s}(0)=\{x \in E:\|x\| \leq s\}$ and $\alpha_{1}, \alpha_{2}, \ldots, \alpha_{N} \in[0,1]$ such that $\sum_{i=1}^{N} \alpha_{i}=1$.

Lemma 2.8. Let $C$ be a closed and convex subset of a uniformly smooth and strictly convex Banach space E. Let $f: C \times C \rightarrow \mathbb{R}$ be a bifunction satisfying (A1), (A2), (A3'), and (A4). Let $r>0$ and $T_{r}: E \rightarrow C$ be a mapping defined by (2.7). Then $T_{\mathrm{r}}$ is closed.

Proof. Let $\left\{x_{n}\right\} \subset E$ converge to $x^{\prime}$ and $\left\{T_{r} x_{n}\right\}$ converge to $\widehat{x}$. To end the conclusion, we need to prove that $T_{r} x^{\prime}=\hat{x}$. Indeed, for each $x_{n}$, Lemma 2.4 shows that there exists a unique $z_{n} \in C$ such that $z_{n}=T_{r} x_{n}$, that is,

$$
f\left(z_{n}, y\right)+\frac{1}{r}\left\langle y-z_{n}, J z_{n}-J x_{n}\right\rangle \geq 0, \quad \forall y \in C
$$


Since $E$ is uniformly smooth, $J$ is continuous on bounded set (note that $\left\{x_{n}\right\}$ and $\left\{z_{n}\right\}$ are both bounded). Taking the limit as $n \rightarrow \infty$ in (2.12), by using (A3'), we get

$$
f(\widehat{x}, y)+\frac{1}{r}\left\langle y-\widehat{x}, J \widehat{x}-J x^{\prime}\right\rangle \geq 0, \quad \forall y \in C
$$

which implies that $T_{r} x^{\prime}=\widehat{x}$. This completes the proof.

\section{Main Results}

Theorem 3.1. Let $C$ be a nonempty closed and convex subset of a uniformly smooth and strictly convex Banach space E which has the Kadec-Klee property. Let $\left\{S_{i}\right\}_{i=1}^{N_{1}}: C \rightarrow C$ be a family of closed quasi- $\phi$-nonexpansive mappings, $\left\{f_{i}\right\}_{i=1}^{N_{2}}: C \times C \rightarrow \mathbb{R}$ a finite family of bifunctions satisfying the conditions $(A 1),(A 2),\left(A 3^{\prime}\right)$, and $(A 4)$, and $\left\{A_{i}\right\}_{i=1}^{N_{3}}: C \rightarrow E^{*}$ a finite family of continuous monotone mappings such that $\mathcal{F}=\left[\bigcap_{i=1}^{N_{1}} F\left(S_{i}\right)\right] \cap\left[\bigcap_{i=1}^{N_{2}} \mathrm{EP}\left(f_{i}\right)\right] \cap\left[\bigcap_{i=1}^{N_{3}} \mathrm{VI}\left(C, A_{i}\right)\right] \neq \emptyset$. Let $\left\{r_{1, i}\right\}_{i=1}^{N_{2}},\left\{r_{2, i}\right\}_{i=1}^{N_{3}} \subset$ $(0, \infty)$. Let $\left\{x_{n}\right\}$ be a sequence generated by the following manner:

$$
\begin{gathered}
x_{1} \in C \text { chosen arbitrarily, } \\
z_{n}=\sum_{i=1}^{N_{1}} \lambda_{1, i} J S_{i} x_{n}, \\
u_{n}=\sum_{i=1}^{N_{2}} \lambda_{2, i} J T_{r_{1, i}} x_{n}, \\
w_{n}=\sum_{i=1}^{N_{3}} \lambda_{3, i} J F_{r_{2, i}} x_{n}, \\
y_{n}=J^{-1}\left(\alpha_{0} J x_{n}+\alpha_{1} z_{n}+\alpha_{2} u_{n}+\alpha_{3} w_{n}\right), \\
C_{n}=\left\{z \in C: \phi\left(v, y_{n}\right) \leq \phi\left(v, x_{n}\right)\right\}, \\
D_{n}=\bigcap_{i=1}^{n} C_{i}, \\
x_{n+1}=\prod_{D_{n}} x_{1}, \quad n \geq 1,
\end{gathered}
$$

where $T_{r_{1, i}}\left(i=1,2, \ldots, N_{2}\right)$ and $F_{r_{2, j}}\left(j=1,2, \ldots, N_{3}\right)$ are defined by (1.11) and (1.12), $\alpha_{0}, \alpha_{1}, \alpha_{2}, \alpha_{3}$ are the real numbers in $(0,1)$ satisfying $\alpha_{0}+\alpha_{1}+\alpha_{2}+\alpha_{3}=1$ and for each $j=1,2,3, \lambda_{j, 1}, \ldots, \lambda_{j, N_{j}}$ are the real numbers in $(0,1)$ satisfying $\sum_{i=1}^{N_{j}} \lambda_{j, i}=1$. Then the sequence $\left\{x_{n}\right\}$ converges strongly to $\Pi_{\mp} x_{1}$, where $\Pi_{\mp}$ is the generalized projection from $E$ onto $\mathcal{F}$.

Proof. First we prove that $D_{n}$ is closed and convex for each $n \geq 1$. From the definition of $C_{n}$, it is obvious that $C_{n}$ is closed. Moreover, since $\phi\left(v, y_{n}\right) \leq \phi\left(v, x_{n}\right)$ is equivalent to $2\left\langle v, J x_{n}-\right.$ $\left.J y_{n}\right\rangle-\left\|x_{n}\right\|^{2}+\left\|y_{n}\right\|^{2} \geq 0$, it follows that $C_{n}$ is convex for each $n \geq 1$. By the definition of $D_{n}$, we can conclude that $D_{n}$ is closed and convex for each $n \geq 1$. 
Next, we prove that $\mathcal{F} \subset D_{n}$ for each $n \geq 1$. From Lemma 2.4 and Remark 2.5, we see that each $T_{r_{1, i}}\left(i=1,2, \ldots, N_{2}\right)$ and $F_{r_{2, j}}\left(j=1,2, \ldots, N_{3}\right)$ are quasi- $\phi$-nonexpansive. Hence, for any $p \in \mathcal{F}$, we have

$$
\begin{aligned}
\phi\left(p, y_{n}\right)= & \phi\left(p, J^{-1}\left(\alpha_{0} J x_{n}+\alpha_{1} z_{n}+\alpha_{2} u_{n}+\alpha_{3} w_{n}\right)\right) \\
= & \|p\|^{2}-2\left\langle p, \alpha_{0} J x_{n}+\alpha_{1} z_{n}+\alpha_{2} u_{n}+\alpha_{3} w_{n}\right\rangle+\left\|\alpha_{0} J x_{n}+\alpha_{1} z_{n}+\alpha_{2} u_{n}+\alpha_{3} w_{n}\right\|^{2} \\
\leq & \|p\|^{2}-2 \alpha_{0}\left\langle p, J x_{n}\right\rangle-2 \alpha_{1}\left\langle p, z_{n}\right\rangle-2 \alpha_{2}\left\langle p, u_{n}\right\rangle \\
& -2 \alpha_{3}\left\langle p, w_{n}\right\rangle+\alpha_{0}\left\|x_{n}\right\|^{2}+\alpha_{1}\left\|z_{n}\right\|^{2}+\alpha_{2}\left\|u_{n}\right\|^{2}+\alpha_{3}\left\|w_{n}\right\|^{2} \\
\leq & \|p\|^{2}-2 \alpha_{0}\left\langle p, J x_{n}\right\rangle-2 \alpha_{1} \sum_{i=1}^{N_{1}} \lambda_{1, i}\left\langle p, J S_{i} x_{n}\right\rangle-2 \alpha_{2} \sum_{i=1}^{N_{2}} \lambda_{2, i}\left\langle p, J T_{r_{1, i}} x_{n}\right\rangle \\
& -2 \alpha_{3} \sum_{i=1}^{N_{3}} \lambda_{3, i}\left\langle p, J F_{r_{2, i}} x_{n}\right\rangle+\alpha_{0}\left\|x_{n}\right\|^{2}+\alpha_{1} \sum_{i=1}^{N_{1}} \lambda_{1, i}\left\|J S_{i} x_{n}\right\|^{2} \\
& +\alpha_{2} \sum_{i=1}^{N_{2}} \lambda_{2, i}\left\|J T_{r_{1, i}} x_{n}\right\|^{2}+\alpha_{3} \sum_{i=1}^{N_{3}} \lambda_{3, i}\left\|J F_{r_{2, i}} x_{n}\right\|^{2} \\
= & \alpha_{0} \phi\left(p, x_{n}\right)+\alpha_{1} \sum_{i=1}^{N_{1}} \lambda_{1, i} \phi\left(p, S_{i} x_{n}\right)+\alpha_{2} \sum_{i=1}^{N_{2}} \lambda_{2, i} \phi\left(p, T_{r_{1, i}} x_{n}\right) \\
& +\alpha_{3} \sum_{i=1}^{N_{3}} \lambda_{3, i} \phi\left(p, F_{r_{2, i}} x_{n}\right) \\
\leq & \alpha_{0} \phi\left(p, x_{n}\right)+\alpha_{1} \sum_{i=1}^{N_{1}} \lambda_{1, i} \phi\left(p, x_{n}\right)+\alpha_{2} \sum_{i=1}^{N_{2}} \lambda_{2, i} \phi\left(p, x_{n}\right) \\
& +\alpha_{3} \sum_{i=1}^{N_{3}} \lambda_{3, i} \phi\left(p, x_{n}\right) \\
= & \phi\left(p, x_{n}\right),
\end{aligned}
$$

which implies that $\mathcal{F} \subset C_{n}$ for each $n \geq 1$. So, it follows from the definition of $D_{n}$ that $F \subset D_{n}$ for each $n \geq 1$. Therefore, the sequence $\left\{x_{n}\right\}$ is well defined. Also, from Lemma 2.2 we see that

$$
\phi\left(x_{n+1}, x_{1}\right)=\phi\left(\Pi_{D_{n}} x_{1}, x_{1}\right) \leq \phi\left(p, x_{1}\right)-\phi\left(p, x_{n+1}\right) \leq \phi\left(p, x_{1}\right),
$$

for each $p \in \mathcal{F}$. This shows that the sequence $\left\{\phi\left(x_{n}, x_{1}\right)\right\}$ is bounded. It follows from (1.4) that the sequence $\left\{x_{n}\right\}$ is also bounded. 
Since $E$ is reflexive, we may, without loss of generality, assume that $x_{n} \rightarrow x^{*}$. Since $D_{n}$ is closed and convex for each $n \geq 1$, we can conclude that $x^{*} \in D_{n}$ for each $n \geq 1$. By the definition of $\left\{x_{n}\right\}$, we see that

$$
\phi\left(x_{n}, x_{1}\right) \leq \phi\left(x^{*}, x_{1}\right)
$$

It follows that

$$
\phi\left(x^{*}, x_{1}\right) \leq \liminf _{n \rightarrow \infty} \phi\left(x_{n}, x_{1}\right) \leq \limsup _{n \rightarrow \infty} \phi\left(x_{n}, x_{1}\right) \leq \phi\left(x^{*}, x_{1}\right) .
$$

This implies that

$$
\lim _{n \rightarrow \infty} \phi\left(x_{n}, x_{1}\right)=\phi\left(x^{*}, x_{1}\right)
$$

Hence, we have $\left\|x_{n}\right\| \rightarrow\left\|x^{*}\right\|$ as $n \rightarrow \infty$. In view of the Kadec-Klee property of $E$, we get that

$$
\lim _{n \rightarrow \infty} x_{n}=x^{*}
$$

By the construction of $D_{n}$, we have that $D_{n+1} \subset D_{n}$ and $x_{n+2}=\Pi_{D_{n+1}} x_{1} \subset D_{n}$. It follows from Lemma 2.2 that

$$
\begin{aligned}
\phi\left(x_{n+2}, x_{n+1}\right) & =\phi\left(x_{n+2}, \Pi_{D_{n}} x_{1}\right) \\
& \leq \phi\left(x_{n+2}, x_{1}\right)-\phi\left(\Pi_{D_{n}} x_{1}, x_{1}\right) \\
& =\phi\left(x_{n+2}, x_{1}\right)-\phi\left(x_{n+1}, x_{1}\right) .
\end{aligned}
$$

Letting $n \rightarrow \infty$, we obtain that $\phi\left(x_{n+2}, x_{n+1}\right) \rightarrow 0$. In view of $x_{n+1} \in D_{n}=\bigcap_{i=1}^{n} C_{n}$, we have $x_{n+1} \in C_{n}$ and hence

$$
\phi\left(x_{n+1}, y_{n}\right) \leq \phi\left(x_{n+1}, x_{n}\right)
$$

It follows that

$$
\lim _{n \rightarrow \infty} \phi\left(x_{n+1}, y_{n}\right)=0
$$

From (1.4), we see that

$$
\left\|y_{n}\right\| \longrightarrow\left\|x^{*}\right\| \quad \text { as } n \rightarrow \infty
$$


Hence,

$$
\left\|J y_{n}\right\| \longrightarrow\left\|J x^{*}\right\| \text { as } n \rightarrow \infty
$$

This implies that the sequence $\left\{J y_{n}\right\}$ is bounded. Note that reflexivity of $E$ implies reflexivity of $E^{*}$. Thus, we may assume that $J y_{n} \rightarrow y \in E^{*}$. Furthermore, reflexivity of $E$ implies that there exists $x \in E$ such that $y=J x$. Then, it follows that

$$
\begin{aligned}
\phi\left(x_{n+1}, y_{n}\right) & =\left\|x_{n+1}\right\|^{2}-2\left\langle x_{n+1}, J y_{n}\right\rangle+\left\|y_{n}\right\|^{2} \\
& =\left\|x_{n+1}\right\|^{2}-2\left\langle x_{n+1}, J y_{n}\right\rangle+\left\|J y_{n}\right\|^{2} .
\end{aligned}
$$

Take lim inf on both sides of (3.13) over $n$ and use weak lower semicontinuity of norm to get that

$$
\begin{aligned}
0 & \geq\left\|x^{*}\right\|^{2}-2\left\langle x^{*}, y\right\rangle+\|y\|^{2} \\
& =\left\|x^{*}\right\|^{2}-2\left\langle x^{*}, J x\right\rangle+\|J x\|^{2} \\
& =\left\|x^{*}\right\|^{2}-2\left\langle x^{*}, J x\right\rangle+\|x\|^{2} \\
& =\phi\left(x^{*}, x\right),
\end{aligned}
$$

which implies that $x^{*}=x$. Hence, $y=J x^{*}$. It follows that $J y_{n} \rightarrow \mathrm{J} x^{*}$. Now, from (3.12) and Kadec-Klee property of $E^{*}$, we obtain that $J y_{n} \rightarrow J x^{*}$ as $n \rightarrow \infty$. Then the demicontinuity of $J^{-1}$ implies that $y_{n} \rightarrow x^{*}$. Now, from (3.11) and the fact that $E$ has the Kadec-Klee property, we obtain that $\lim _{n \rightarrow \infty} y_{n}=x^{*}$. Note that

$$
\left\|x_{n}-y_{n}\right\| \leq\left\|x_{n}-x^{*}\right\|+\left\|x^{*}-y_{n}\right\|
$$

It follows that

$$
\lim _{n \rightarrow \infty}\left\|x_{n}-y_{n}\right\|=0
$$

Since $J$ is uniformly norm-to-norm continuous on any bounded sets, we have

$$
\lim _{n \rightarrow \infty}\left\|J x_{n}-J y_{n}\right\|=0
$$


Since $E$ is uniformly smooth, we know that $E^{*}$ is uniformly convex. In view of Lemma 2.7, we see that, for any $p \in \mathcal{F}$,

$$
\begin{aligned}
\phi\left(p, y_{n}\right)= & \phi\left(p, J^{-1}\left(\alpha_{0} J x_{n}+\alpha_{1} z_{n}+\alpha_{2} u_{n}+\alpha_{3} w_{n}\right)\right) \\
= & \|p\|^{2}-2\left\langle p, \alpha_{0} J x_{n}+\alpha_{1} z_{n}+\alpha_{2} u_{n}+\alpha_{3} w_{n}\right\rangle+\left\|\alpha_{0} J x_{n}+\alpha_{1} z_{n}+\alpha_{2} u_{n}+\alpha_{3} w_{n}\right\|^{2} \\
\leq & \|p\|^{2}-2\left\langle p, \alpha_{0} J x_{n}+\alpha_{1} z_{n}+\alpha_{2} u_{n}+\alpha_{3} w_{n}\right\rangle+\alpha_{0}\left\|x_{n}\right\|^{2}+\alpha_{1} \sum_{i=1}^{N_{1}} \lambda_{1, i}\left\|S_{i} x_{n}\right\|^{2} \\
& +\alpha_{2} \sum_{i=1}^{N_{2}} \lambda_{2, i}\left\|T_{r_{1, i}} x_{n}\right\|^{2}+\alpha_{3} \sum_{i=1}^{N_{3}} \lambda_{3, i}\left\|F_{r_{2, i}} x_{n}\right\|^{2}-\alpha_{0} \alpha_{1} \lambda_{1,1} g\left(\left\|J x_{n}-J S_{1} x_{n}\right\|\right) \\
= & \alpha_{0} \phi\left(p, x_{n}\right)+\alpha_{1} \sum_{i=1}^{N_{1}} \lambda_{1, i} \phi\left(p, S_{i} x_{n}\right)+\alpha_{2} \sum_{i=1}^{N_{2}} \lambda_{2, i} \phi\left(p, T_{r_{1, i}} x_{n}\right) \\
& +\alpha_{3} \sum_{i=1}^{N_{3}} \lambda_{3, i} \phi\left(p, F_{r_{2, i}} x_{n}\right)-\alpha_{0} \alpha_{1} \lambda_{1,1} g\left(\left\|J x_{n}-J S_{1} x_{n}\right\|\right) \\
\leq & \alpha_{0} \phi\left(p, x_{n}\right)+\alpha_{1} \sum_{i=1}^{N_{1}} \lambda_{1, i} \phi\left(p, x_{n}\right)+\alpha_{2} \sum_{i=1}^{N_{2}} \lambda_{2, i} \phi\left(p, x_{n}\right)+\alpha_{3} \sum_{i=1}^{N_{3}} \lambda_{3, i} \phi\left(p, x_{n}\right) \\
& -\alpha_{0} \alpha_{1} \lambda_{1,1} g\left(\left\|J x_{n}-J S_{1} x_{n}\right\|\right) \\
= & \phi\left(p, x_{n}\right)-\alpha_{0} \alpha_{1} \lambda_{1,1} g\left(\left\|J x_{n}-J S_{1} x_{n}\right\|\right) .
\end{aligned}
$$

It follows that

$$
\alpha_{0} \alpha_{1} \lambda_{1,1} g\left(\left\|J x_{n}-J S_{1} x_{n}\right\|\right) \leq \phi\left(p, x_{n}\right)-\phi\left(p, y_{n}\right)
$$

Note that

$$
\begin{aligned}
\phi\left(p, x_{n}\right)-\phi\left(p, y_{n}\right) & =\left\|x_{n}\right\|^{2}-\left\|y_{n}\right\|^{2}-2\left\langle p, J x_{n}-J y_{n}\right\rangle \\
& \leq\left\|x_{n}-y_{n}\right\|\left(\left\|x_{n}\right\|+\left\|y_{n}\right\|\right)+2\|p\|\left\|J x_{n}-J y_{n}\right\|
\end{aligned}
$$

It follows from (3.16) and (3.17) that

$$
\phi\left(p, x_{n}\right)-\phi\left(p, y_{n}\right) \longrightarrow 0 \quad \text { as } n \longrightarrow \infty
$$

By (3.19), (3.21), and $\alpha_{0} \alpha_{1} \lambda_{1,1}>0$, we have

$$
g\left(\left\|J x_{n}-J S_{1} x_{n}\right\|\right) \longrightarrow 0 \quad \text { as } n \longrightarrow \infty
$$


Fixed Point Theory and Applications

It follows from the property of $g$ that

$$
\left\|J x_{n}-J S_{1} x_{n}\right\| \longrightarrow 0 \text { as } n \longrightarrow \infty \text {. }
$$

Since $x_{n} \rightarrow x^{*}$ as $n \rightarrow \infty$ and $J: E \rightarrow E^{*}$ is demicontinuous, we obtain that $J x_{n} \rightarrow J x^{*} \in E^{*}$. Note that

$$
\left|\left\|J x_{n}\right\|-\left\|J x^{*}\right\|\right|=\left|\left\|x_{n}\right\|-\left\|x^{*}\right\|\right| \leq\left\|x_{n}-x^{*}\right\| .
$$

This implies that

$$
\lim _{n \rightarrow \infty}\left\|J x_{n}\right\|=\left\|J x^{*}\right\|
$$

Since $E^{*}$ enjoys the Kadec-Klee property, we see that

$$
\lim _{n \rightarrow \infty}\left\|J x_{n}-J x^{*}\right\|=0
$$

Note that

$$
\left\|J S_{1} x_{n}-J x^{*}\right\| \leq\left\|J S_{1} x_{n}-J x_{n}\right\|+\left\|J x_{n}-J x^{*}\right\|
$$

From (3.23) and (3.26), we arrive at

$$
\lim _{n \rightarrow \infty}\left\|J S_{1} x_{n}-J x^{*}\right\|=0
$$

Note that $J^{-1}: E^{*} \rightarrow E$ is demicontinuous. It follows that $S_{1} x_{n} \rightarrow x^{*}$. On the other hand, since

$$
\left|\left\|S_{1} x_{n}\right\|-\left\|x^{*}\right\|\right|=\left|\left\|J S_{1} x_{n}\right\|-\left\|J x^{*}\right\|\right| \leq\left\|J S_{1} x_{n}-J x^{*}\right\|,
$$

by (3.28) we conclude that $\left\|S_{1} x_{n}\right\| \rightarrow\left\|x^{*}\right\|$ as $n \rightarrow \infty$. Since $E$ enjoys the Kadec-Klee property, we obtain that

$$
\lim _{n \rightarrow \infty}\left\|S_{1} x_{n}-x^{*}\right\|=0
$$

By repeating (3.18)-(3.30), we also can get

$$
\begin{aligned}
& \lim _{n \rightarrow \infty}\left\|S_{i} x_{n}-x^{*}\right\|=0, \quad i=2, \ldots, N_{1}, \\
& \lim _{n \rightarrow \infty}\left\|T_{r_{1, i}} x_{n}-x^{*}\right\|=0, \quad i=1, \ldots, N_{2}, \\
& \lim _{n \rightarrow \infty}\left\|F_{r_{2, i}} x_{n}-x^{*}\right\|=0, \quad i=1, \ldots, N_{3} .
\end{aligned}
$$


Since each $S_{i}$ is closed, by (3.30) and (3.31) we conclude that $S_{i} x^{*}=x^{*}$, that is, $x^{*} \in F\left(S_{i}\right), i=1,2, \ldots, N_{1}$. On the other hand, Lemma 2.4, Remark 2.5, and Lemma 2.8 show that $T_{r_{1, i}}\left(i=1,2, \ldots, N_{2}\right)$ and $F_{r_{2, i}}\left(i=1,2, \ldots, N_{3}\right)$ are closed. So, by (3.32) and (3.33) we have $T_{r_{1, i}} x^{*}=x^{*}\left(i=1,2, \ldots, N_{2}\right)$ and $F_{r_{2, i}} x^{*}=x^{*}\left(i=1,2, \ldots, N_{3}\right)$. Now, it follows from Lemma 2.4 and Remark 2.5 that $F\left(T_{r_{1, i}}\right)=\operatorname{EP}\left(f_{i}\right)\left(i=1,2, \ldots, N_{2}\right)$ and $F\left(F_{r_{2, i}}\right)=\operatorname{VI}\left(C, A_{i}\right)$ $\left(i=1,2, \ldots, N_{3}\right)$. Hence, $x^{*} \in \operatorname{EP}\left(f_{i}\right)\left(i=1,2, \ldots, N_{2}\right)$ and $x^{*} \in \operatorname{VI}\left(C, A_{i}\right)\left(i=1,2, \ldots, N_{3}\right)$. Therefore, $x^{*} \in \mathcal{F}$.

Finally, we prove that $x^{*}=\Pi_{\mp} x_{1}$. From $x_{n+1}=\Pi_{D_{n}} x_{1}$, by Lemma 2.1, we see that

$$
\left\langle x_{n+1}-p, J x_{1}-J x_{n+1}\right\rangle \geq 0, \quad \forall p \in D_{n} .
$$

Since $\mathcal{F} \subset D_{n}$ for each $n \geq 1$, we have

$$
\left\langle x_{n+1}-p, J x_{1}-J x_{n+1}\right\rangle \geq 0, \quad \forall p \in \mathcal{F} .
$$

Letting $n \rightarrow \infty$ in (3.35), we see that

$$
\left\langle x^{*}-p, J x_{1}-J p\right\rangle \geq 0, \quad \forall p \in \mathcal{F} .
$$

In view of Lemma 2.1, we can obtain that $x^{*}=\Pi_{\mp} x_{1}$. This completes the proof.

Remark 3.2. Obviously, the proof process of $x^{*} \in\left[\bigcap_{i=1}^{N_{2}} \operatorname{EP}\left(f_{i}\right)\right] \cap\left[\bigcap_{i=1}^{N_{3}} \operatorname{VI}\left(C, A_{i}\right)\right]$ is simple since we replace the condition (A3) with (A3') which is such that $T_{r_{1, i}}$ and $F_{r_{2, j}}\left(i=1,2, \ldots, N_{2}, j=\right.$ $\left.1,2, \ldots, N_{3}\right)$ are closed. In fact, although the condition (A3') is stronger than (A3), it is not easier to verify the condition (A3) than verify the condition (A3'). Hence, from this point, the condition (A3') is acceptable. On the other hand, the definition of $D_{n}$ is of some interest.

If $S_{i}=S$ for each $i=1,2, \ldots, N_{1}, f_{i}=f$ for each $i=1,2, \ldots, N_{2}$ and $A_{i}=A$ for each $i=1,2, \ldots, N_{3}$, then Theorem 3.1 reduces to the following result.

Corollary 3.3. Let $C$ be a nonempty closed and convex subset of a uniformly smooth and strictly convex Banach space E which has the Kadec-Klee property. Let $S: C \rightarrow C$ be a closed quasi- $\phi-$ nonexpansive mapping, $f: C \times C \rightarrow \mathbb{R}$ a bifunction satisfying the conditions (A1), (A2), (A3'), and $(A 4)$ and $A: C \rightarrow E^{*}$ a continuous monotone mapping such that $\mathcal{F}=F(S) \cap \operatorname{EP}(f) \cap \operatorname{VI}(C, A) \neq \emptyset$. Let $r_{1}, r_{2} \subset(0, \infty)$. Let $\left\{x_{n}\right\}$ be a sequence defined by the following manner:

$$
\begin{gathered}
x_{1} \in C \text { chosen arbitrarily, } \\
y_{n}=J^{-1}\left(\alpha_{0} J x_{n}+\alpha_{1} J S x_{n}+\alpha_{2} J T_{r_{1}} x_{n}+\alpha_{3} J F_{r_{2}} x_{n}\right), \\
C_{n}=\left\{z \in C: \phi\left(v, y_{n}\right) \leq \phi\left(v, x_{n}\right)\right\}, \\
D_{n}=\bigcap_{i=1}^{n} C_{i} \\
x_{n+1}=\prod_{D_{n}} x_{1}, \quad n \geq 1
\end{gathered}
$$


where $T_{r_{1}}$ and $F_{r_{2}}$ are defined by (1.11) and (1.12) with $r_{1, i}=r_{1}\left(i=1,2, \ldots, N_{2}\right)$ and $r_{2, j}=r_{2}(j=$ $\left.1,2, \ldots, N_{3}\right), \alpha_{0}, \alpha_{1}, \alpha_{2}, \alpha_{3}$ are the real numbers in $(0,1)$ satisfying $\alpha_{0}+\alpha_{1}+\alpha_{2}+\alpha_{3}=1$. Then the sequence $\left\{x_{n}\right\}$ converges strongly to $P_{\mp} x_{1}$, where $\Pi_{\mp}$ is the generalized projection from $E$ onto $F$.

Corollary 3.4. Let $C$ be a nonempty closed and convex subset of a Hilbert space H. Let $\left\{S_{i}\right\}_{i=1}^{N_{1}}: C \rightarrow$ $C$ be a family of closed quasi-nonexpansive mappings, $\left\{f_{i}\right\}_{i=1}^{N_{2}}: C \times C \rightarrow \mathbb{R}$ a finite family of bifunctions satisfying the conditions (A1)-(A4), and $\left\{A_{i}\right\}_{i=1}^{N_{3}}: C \rightarrow H$ a finite family of continuous monotone mappings such that $\mathcal{F}=\left[\bigcap_{i=1}^{N_{1}} F\left(S_{i}\right)\right] \cap\left[\bigcap_{i=1}^{N_{2}} \mathrm{EP}\left(f_{i}\right)\right] \cap\left[\bigcap_{i=1}^{N_{3}} \mathrm{VI}\left(C, A_{i}\right)\right] \neq \emptyset$. Let $\left\{r_{1, i}\right\}_{i=1}^{N_{2}},\left\{r_{2, i}\right\}_{i=1}^{N_{3}} \subset$ $(0, \infty)$. Define a sequence $\left\{x_{n}\right\}$ by the following manner:

$$
\begin{gathered}
x_{1} \in C \text { chosen arbitrarily, } \\
z_{n}=\sum_{i=1}^{N_{1}} \lambda_{1, i} S_{i} x_{n}, \\
u_{n}=\sum_{i=1}^{N_{2}} \lambda_{2, i} T_{r_{1, i}} x_{n}, \\
w_{n}=\sum_{i=1}^{N_{3}} \lambda_{3, i} F_{r_{2, i}} x_{n}, \\
y_{n}=\left(\alpha_{0} x_{n}+\alpha_{1} z_{n}+\alpha_{2} u_{n}+\alpha_{3} w_{n}\right), \\
C_{n}=\left\{z \in C:\left\|v-y_{n}\right\| \leq\left\|v-x_{n}\right\|\right\}, \\
D_{n}=\bigcap_{i=1}^{n} C_{i}, \\
x_{n+1}=P_{D_{n}} x_{1}, \quad n \geq 1,
\end{gathered}
$$

where $\left\{T_{r_{1, i}}\right\}_{i=1}^{N_{2}}$ and $\left\{F_{r_{1, i}}\right\}_{i=1}^{N_{3}}$ are defined by (1.11) and (1.12) with $J=I$ ( $I$ is the identity mapping), $\alpha_{0}, \alpha_{1}, \alpha_{2}, \alpha_{3}$ are the real numbers in $(0,1)$ satisfying $\alpha_{0}+\alpha_{1}+\alpha_{2}+\alpha_{3}=1$ and for each $j=1,2,3$, $\lambda_{j, 1}, \ldots, \lambda_{j, N_{j}}$ are the real numbers in $(0,1)$ satisfying $\sum_{i=1}^{N_{j}} \lambda_{j, i}=1$. Then the sequence $\left\{x_{n}\right\}$ converges strongly to $P_{\mp} x_{1}$, where $P_{\mp}$ is the projection from $\mathrm{H}$ onto $₹$.

Proof. By the proof of Theorem 3.1, we have $x_{n} \rightarrow x^{*}$ as $n \rightarrow \infty$,

$$
\begin{aligned}
& \lim _{n \rightarrow \infty}\left\|S_{i} x_{n}-x_{n}\right\|=0, \quad i=1,2, \ldots, N_{1}, \\
& \lim _{n \rightarrow \infty}\left\|T_{r_{1, i}} x_{n}-x_{n}\right\|=0, \quad i=1,2, \ldots, N_{2}, \\
& \lim _{n \rightarrow \infty}\left\|F_{r_{2, i}} x_{n}-x_{n}\right\|=0, \quad i=1,2, \ldots, N_{3} .
\end{aligned}
$$

Since each $S_{i}$ is closed, we can conclude that $x^{*} \in F\left(S_{i}\right), i=1,2, \ldots, N_{1}$. Note that in a Hilbert space, a firmly-nonexpansive mapping is also nonexpansive. Hence, $T_{r_{1}, i}$ and $F_{r_{2, j}}$ are nonexpansive for each $i=1,2, \ldots, N_{2}$ and $j=1,2, \ldots, N_{3}$. By demiclosed principle, we can conclude that $x^{*} \in F\left(T_{r 1, i}\right)=\mathrm{EP}\left(f_{i}\right)$ and $x^{*} \in F\left(F_{r 2, i}\right)=\mathrm{VI}\left(C, A_{j}\right)$ for each $i=1,2, \ldots, N_{2}$ and $j=1,2, \ldots, N_{3}$. That is, $x^{*} \in \mathcal{F}$. Then by the final part of proof of Theorem 3.1, we have $x_{n} \rightarrow x^{*}=P_{\mp} x_{1}$. This completes the proof. 
Let $H$ be a Hilbert space and $C$ a nonempty closed and convex subset of $H$. A mapping $T: C \rightarrow H$ is called a pseudocontraction if for all $x, y \in C$,

$$
\|T x-T y\|^{2} \leq\|x-y\|^{2}+\|(I-T) x-(I-T) y\|^{2},
$$

or equivalently,

$$
\langle(I-T) x-(I-T) y, x-y\rangle \geq 0
$$

Let $A=I-T$, where $T: C \rightarrow H$ is a pseudocontraction. Then $A$ is a monotone mapping and $A^{-1}(0)=F(T)$. Moreover, $F(T)=\operatorname{VI}(C, A)$. Indeed, it is easy to see that $F(T) \subset$ $\mathrm{VI}(C, A)$. Let $u \in \mathrm{VI}(C, A)$. We have

$$
\langle v-u, A u\rangle \geq 0, \quad \text { i.e., }\langle v-u,(I-T) u\rangle \geq 0,
$$

for all $v \in C$. Take $v=T u$. Then we have $\langle T u-u,(I-T) u\rangle \geq 0$. That is, $-\|u-T u\|^{2} \geq 0$. This shows that $u=T u$, which implies that $\operatorname{VI}(C, A) \subset F(T)$. So, $F(T)=\operatorname{VI}(C, A)$. Based this, we have following result.

Corollary 3.5. Let $C$ be a nonempty closed and convex subset of a Hilbert space H. Let $\left\{S_{i}\right\}_{i=1}^{N_{1}}$ : $C \rightarrow C$ be a family of closed quasi-nonexpansive mappings, $\left\{f_{i}\right\}_{i=1}^{N_{2}}: C \times C \rightarrow \mathbb{R}$ a finite family of bifunctions satisfying the conditions (A1)-(A4), and $\left\{T_{i}\right\}_{i=1}^{N_{3}}: C \rightarrow H$ a finite family of continuous pseudocontractions such that $\mathcal{F}=\left[\bigcap_{i=1}^{N_{1}} F\left(S_{i}\right)\right] \cap\left[\bigcap_{i=1}^{N_{2}} \operatorname{EP}\left(f_{i}\right)\right] \cap\left[\bigcap_{i=1}^{N_{3}} F\left(T_{i}\right)\right] \neq \emptyset$. Let $\left\{r_{1, i}\right\}_{i=1}^{N_{2}},\left\{r_{2, i}\right\}_{i=1}^{N_{3}} \subset(0, \infty)$. Define a sequence $\left\{x_{n}\right\}$ by the following manner:

$$
\begin{gathered}
x_{1} \in C \text { chosen arbitrarily, } \\
z_{n}=\sum_{i=1}^{N_{1}} \lambda_{1, i} S_{i} x_{n}, \\
u_{n}=\sum_{i=1}^{N_{2}} \lambda_{2, i} T_{r_{1, i}} x_{n}, \\
w_{n}=\sum_{i=1}^{N_{3}} \lambda_{3, i} F_{r_{2, i}} x_{n}, \\
y_{n}=\left(\alpha_{0} x_{n}+\alpha_{1} z_{n}+\alpha_{2} u_{n}+\alpha_{3} w_{n}\right), \\
C_{n}=\left\{z \in C:\left\|v-y_{n}\right\| \leq\left\|v-x_{n}\right\|\right\}, \\
D_{n}=\bigcap_{i=1}^{n} C_{i}, \\
x_{n+1}=P_{D_{n}} x_{1}, \quad n \geq 1,
\end{gathered}
$$


where $\left\{T_{r_{1, i}}\right\}_{i=1}^{N_{2}}$ are defined by (1.11) with $J=I$ and $F_{r_{2, i}}$ is defined by

$$
F_{r_{2, i}}(x)=\left\{z \in C:\left\langle y-x,\left(I-T_{i}\right) x\right\rangle+\frac{1}{r_{2, i}}\langle y-z, z-x\rangle \geq 0 \forall y \in C\right\}, \quad i=1,2, \ldots, N_{3},
$$

$\alpha_{0}, \alpha_{1}, \alpha_{2}, \alpha_{3}$ are the real numbers in $(0,1)$ satisfying $\alpha_{0}+\alpha_{1}+\alpha_{2}+\alpha_{3}=1$ and for each $j=1,2,3$, $\lambda_{j, 1}, \ldots, \lambda_{j, N_{j}}$ are the real numbers in $(0,1)$ satisfying $\sum_{i=1}^{N_{j}} \lambda_{j, i}=1$. Then the sequence $\left\{x_{n}\right\}$ converges strongly to $P_{\mp} x_{1}$, where $P_{\mp}$ is the projection from $\mathrm{H}$ onto $₹$.

If $S_{i}=S, f_{j}=f$, and $T_{k}=T$ for each $i=1,2, \ldots, N_{1}, j=1,2, \ldots, N_{2}$, and $k=1,2, \ldots, N_{3}$, then Corollary 3.5 reduced the following result.

Corollary 3.6. Let $C$ be a nonempty closed and convex subset of a Hilbert space $H$. Let $S: C \rightarrow C$ be a closed quasi-nonexpansive mapping, $f: C \times C \rightarrow \mathbb{R}$ a bifunction satisfying the conditions (A1)$(A 4)$, and $T: C \rightarrow H$ a continuous pseudocontraction such that $\mathcal{F}=F(S) \cap \operatorname{EP}(f)] \cap F(T) \neq \emptyset$. Let $r_{1}, r_{2} \subset(0, \infty)$. Define a sequence $\left\{x_{n}\right\}$ by the following manner:

$$
\begin{gathered}
x_{1} \in C \text { chosen arbitrarily, } \\
y_{n}=J^{-1}\left(\alpha_{0} x_{n}+\alpha_{1} S x_{n}+\alpha_{2} T_{r_{1}} x_{n}+\alpha_{3} F_{r_{2}} x_{n}\right), \\
C_{n}=\left\{z \in C:\left\|v-y_{n}\right\| \leq\left\|v-x_{n}\right\|\right\}, \\
D_{n}=\bigcap_{i=1}^{n} C_{i}, \\
x_{n+1}=P_{D_{n}} x_{1}, \quad n \geq 1,
\end{gathered}
$$

where $T_{r_{1}}$ is defined by (1.11) with $J=I$ and $r_{1, i}=r_{1}\left(i=1,2, \ldots, N_{2}\right), F_{r_{2}}$ is defined by (3.44) $r_{2, j}=$ $r_{2}\left(j=1,2, \ldots, N_{3}\right)$, and $\alpha_{0}, \alpha_{1}, \alpha_{2}, \alpha_{3}$ are the real numbers in $(0,1)$ satisfying $\alpha_{0}+\alpha_{1}+\alpha_{2}+\alpha_{3}=1$. Then the sequence $\left\{x_{n}\right\}$ converges strongly to $P_{\mp} x_{1}$, where $P_{\mp}$ is the projection from $H$ onto $F$.

\section{Acknowledgment}

This work was supported by the Natural Science Foundation of Hebei Province (A2010001482).

\section{References}

[1] Y. I. Alber, "Metric and generalized projection operators in Banach spaces: properties and applications," in Theory and Applications of Nonlinear Operators of Accretive and Monotone Type, vol. 178 of Lecture Notes in Pure and Appl. Math., pp. 15-50, Dekker, New York, NY, USA, 1996.

[2] Ya. I. Alber and S. Reich, "An iterative method for solving a class of nonlinear operator equations in Banach spaces," Panamerican Mathematical Journal, vol. 4, no. 2, pp. 39-54, 1994.

[3] I. Cioranescu, Geometry of Banach Spaces, Duality Mappings and Nonlinear Problems, vol. 62 of Mathematics and Its Applications, Kluwer Academic Publishers, Dordrecht, The Netherlands, 1990.

[4] W. Takahashi, Nonlinear Functional Analysis, Yokohama Publishers, Yokohama, Japan, 2000, Fixed point theory and Its application.

[5] S. Reich, "A weak convergence theorem for the alternating method with Bregman distances," in Theory and Applications of Nonlinear Operators of Accretive and Monotone Type, A. G. Kartsatos, Ed., vol. 178 of Lecture Notes in Pure and Appl. Math., pp. 313-318, Dekker, New York, NY, USA, 1996. 
[6] D. Butnariu, S. Reich, and A. J. Zaslavski, "Asymptotic behavior of relatively nonexpansive operators in Banach spaces," Journal of Applied Analysis, vol. 7, no. 2, pp. 151-174, 2001.

[7] D. Butnariu, S. Reich, and A. J. Zaslavski, "Weak convergence of orbits of nonlinear operators in reflexive Banach spaces," Numerical Functional Analysis and Optimization, vol. 24, no. 5-6, pp. 489-508, 2003.

[8] Y. Censor and S. Reich, "Iterations of paracontractions and firmly nonexpansive operators with applications to feasibility and optimization," Optimization, vol. 37, no. 4, pp. 323-339, 1996.

[9] H. Zhou, G. Gao, and B. Tan, "Convergence theorems of a modified hybrid algorithm for a family of quasi- $\phi$-asymptotically nonexpansive mappings," Journal of Applied Mathematics and Computing, vol. 32, no. 2, pp. 453-464, 2010.

[10] S.-y. Matsushita and W. Takahashi, "A strong convergence theorem for relatively nonexpansive mappings in a Banach space," Journal of Approximation Theory, vol. 134, no. 2, pp. 257-266, 2005.

[11] L.-C. Ceng and J.-C. Yao, "Hybrid viscosity approximation schemes for equilibrium problems and fixed point problems of infinitely many nonexpansive mappings," Applied Mathematics and Computation, vol. 198, no. 2, pp. 729-741, 2008.

[12] L.-C. Ceng, S. Al-Homidan, Q. H. Ansari, and J.-C. Yao, "An iterative scheme for equilibrium problems and fixed point problems of strict pseudo-contraction mappings," Journal of Computational and Applied Mathematics, vol. 223, no. 2, pp. 967-974, 2009.

[13] L. C. Ceng, A. Petruşel, and J. C. Yao, "Iterative approaches to solving equilibrium problems and fixed point problems of infinitely many nonexpansive mappings," Journal of Optimization Theory and Applications, vol. 143, no. 1, pp. 37-58, 2009.

[14] S.-S. Chang, Y. J. Cho, and J. K. Kim, "Approximation methods of solutions for equilibrium problem in Hilbert spaces," Dynamic Systems and Applications, vol. 17, no. 3-4, pp. 503-513, 2008.

[15] S.-S. Chang, H. W. Joseph Lee, and C. K. Chan, "A new method for solving equilibrium problem fixed point problem and variational inequality problem with application to optimization," Nonlinear Analysis: Theory, Methods \& Applications, vol. 70, no. 9, pp. 3307-3319, 2009.

[16] P. Kumam and P. Katchang, "A viscosity of extragradient approximation method for finding equilibrium problems, variational inequalities and fixed point problems for nonexpansive mappings," Nonlinear Analysis: Hybrid Systems, vol. 3, no. 4, pp. 475-486, 2009.

[17] A. Moudafi, "Weak convergence theorems for nonexpansive mappings and equilibrium problems," Journal of Nonlinear and Convex Analysis, vol. 9, no. 1, pp. 37-43, 2008.

[18] S. Plubtieng and R. Punpaeng, "A new iterative method for equilibrium problems and fixed point problems of nonexpansive mappings and monotone mappings," Applied Mathematics and Computation, vol. 197, no. 2, pp. 548-558, 2008.

[19] X. Qin, Y. J. Cho, and S. M. Kang, "Viscosity approximation methods for generalized equilibrium problems and fixed point problems with applications," Nonlinear Analysis: Theory, Methods $\mathcal{E}$ Applications, vol. 72, no. 1, pp. 99-112, 2010.

[20] X. Qin, S. Y. Cho, and S. M. Kang, "Strong convergence of shrinking projection methods for quasi- $\phi$ nonexpansive mappings and equilibrium problems," Journal of Computational and Applied Mathematics, vol. 234, no. 3, pp. 750-760, 2010.

[21] S. Takahashi and W. Takahashi, "Viscosity approximation methods for equilibrium problems and fixed point problems in Hilbert spaces," Journal of Mathematical Analysis and Applications, vol. 331, no. 1, pp. 506-515, 2007.

[22] W. Takahashi and K. Zembayashi, "Strong and weak convergence theorems for equilibrium problems and relatively nonexpansive mappings in Banach spaces," Nonlinear Analysis: Theory, Methods $\mathcal{E}$ Applications, vol. 70, no. 1, pp. 45-57, 2009.

[23] S. Wang, G. Marino, and F. Wang, "Strong convergence theorems for a generalized equilibrium problem with a relaxed monotone mapping and a countable family of nonexpansive mappings in a Hilbert space," Fixed Point Theory and Applications, vol. 2010, Article ID 230304, 22 pages, 2010.

[24] Y. J. Cho, S. Wang, and X. Qin, "A new iterative method for solving equilibrium problems and fixed point problems for infinite family of nonexpansive mappings," Fixed Point Theory and Applications, vol. 2010, Article ID 165098, 18 pages, 2010.

[25] H. Zegeye and N. Shahzad, "A hybrid scheme for finite families of equilibrium, variational inequality and fixed point problems," Nonlinear Analysis: Theory, Methods E Applications, vol. 74, no. 1, pp. 263272, 2011.

[26] H. K. Xu, "Inequalities in Banach spaces with applications," Nonlinear Analysis: Theory, Methods $\mathcal{E}$ Applications, vol. 16, no. 12, pp. 1127-1138, 1991. 